\title{
BMJ Open An international qualitative study exploring patients' experiences of cutaneous leishmaniasis: study set-up and protocol
}

\author{
Astrid Christine Erber, ${ }^{1,2}$ Byron Arana, ${ }^{3}$ Issam Bennis, ${ }^{4,5}$ Afif Ben Salah,, \\ Aicha Boukthir, ${ }^{6}$ Maria del Mar Castro Noriega, ${ }^{8,9}$ Mamoudou Cissé, ${ }^{10}$ \\ Gláucia Fernandes Cota, ${ }^{11}$ Farhad Handjani, ${ }^{12}$ Mairie Guizaw Kebede, ${ }^{13}$ \\ Trudie Lang, ${ }^{1}$ Liliana López Carvajal, ${ }^{14}$ Kevin Marsh, ${ }^{1}$ Dalila Martinez Medina, ${ }^{15,16}$ \\ Emma Plugge, ${ }^{1}$ Piero Olliaro ${ }^{1,17}$
}

To cite: Erber AC, Arana B, Bennis I, et al. An international qualitative study exploring patients' experiences of cutaneous leishmaniasis: study set-up and protocol. BMJ Open 2018;8:e021372. doi:10.1136/ bmjopen-2017-021372

- Prepublication history and additional material for this paper are available online. To view please visit the journal (http:// dx.doi.org/10.1136/bmjopen2017-021372).

Received 26 December 2017 Revised 18 April 2018 Accepted 4 May 2018

Check for updates

For numbered affiliations see end of article.

Correspondence to Professor Piero Olliaro; olliarop@who.int

\section{ABSTRACT}

Introduction Lack of investments in drug development, lack of standardisation of clinical trials and the complexity of disease presentations contribute to the current lack of effective, safe and adapted treatments for cutaneous leishmaniasis (CL). One aspect concerns outcomes affecting patients' quality of life (QoL): these are hardly assessed in trials, despite potential functional and/or aesthetic impairment caused by $\mathrm{CL}$, which typically affects disadvantaged and vulnerable people living in rural areas. Here, we describe the approach used to bring perspectives of patients with $\mathrm{CL}$ into designing and assessing treatments.

Methods and analysis This international qualitative study uses interviews with patients to explore their experiences with CL to (1) elicit outcomes and eligibility criteria for clinical trials important to them and (2) to better understand their needs and views about the disease and their requirements and expectations from treatment. Here, we describe the set-up of this collaborative study and the protocol. Data collection is ongoing. The protocol includes study design, preparation, conduct and analysis of individual interviews with approximately 80 patients in seven countries (Burkina Faso, Brazil, two sites in Colombia, Iran, Morocco, Peru and Tunisia) where CL is prevalent. Principal investigators and sites were selected through an open call, and two workshops were organised for protocol development and training in conduct and analysis of qualitative health research. Patient recruitment aims at covering a maximum variation of experiences. Transcripts will be analysed to identify outcomes and eligibility criteria as well as further topics that are expected to emerge from the interviews, such as direct and indirect costs related to $\mathrm{CL}$, its psychological impact, preferred modes of drug administration and traditional treatments.

Ethics and dissemination The study received ethical approval by the responsible committees of each of the participating institutions. Findings will be disseminated through publication in peer-reviewed journals, scientific meetings and to participants and their communities.

\section{Strengths and limitations of this study}

- A sampling framework guided by clinical parameters was designed for the entire study and implemented at individual sites with the aim of capturing a spectrum of patient experiences in these regions that is as broad as possible.

- The conduct of interviews in six different languages, corresponding to patients' mother tongues, requires strong reliance on good translation and capturing of additional information, but might limit interpretation.

- Due to the multicentre nature of this study, the analysis of all interviews for outcomes and eligibility criteria will be conducted by two researchers who have not been involved in the interviews, which is unusual in qualitative research. However, feedback will be sought from the local participating experts in order to validate the preliminary individual site results and on the overall results.

- Results are expected to reflect the complex disease spectrum of cutaneous leishmaniasis as well as the personal and cultural context of patients, raising the possibility that some results might be limited to specific regions.

\section{INTRODUCTION}

Deficiencies of clinical trials for CL interventions Cutaneous leishmaniasis (CL) is the most common of the forms of disease caused by protozoan parasites belonging to the genus Leishmania, which include also visceral leishmaniasis (VL) or kala-azar (with post-kala azar dermal leishmaniasis (PKDL)) and mucocutaneous leishmaniasis (MCL). An estimated 0.7-1.3 million new cases of CL occur worldwide annually, $95 \%$ of which are reported in the Americas, the Mediterranean basin and the Middle East and Central Asia and over two-thirds in six countries: Afghanistan, 
Algeria, Brazil, Colombia, the Islamic Republic of Iran and the Syrian Arab Republic. ${ }^{12}$

While for convenience referred to as a single entity, CL is in reality shorthand for a range of cutaneous manifestations caused by different species of Leishmania. Based on where they occur, they are divided in two broad groups: the old-world and the new-world cutaneous leishmaniasis (OWCL and NWCL). Although not a fatal disease, CL causes visible lesions on exposed parts of the body, including ulcers, nodules, plaques and a variety of other less frequent manifestations, which can be distressing, discomforting and potentially leaving life-long scars.

Many treatments have been tried, but one that is effective for all clinical forms of the disease, safe and easy to administer has yet to be found. This is a direct consequence of the lack of investments in developing new treatments for this condition, but it is also partly related to the deficiencies in design and conduct of studies and the lack of standardised clinical trial methodologies, as pointed out by systematic reviews. ${ }^{3-8}$

While progress has been made in reaching consensus on clinical trial methodologies in CL, ${ }^{910}$ patient's views have not yet been genuinely considered, especially regarding why treatment is required and what outcomes are sought. In particular, outcomes affecting patients' quality of life (QoL) are rarely reported in clinical studies: of the 48 studies included in systematic reviews of trials conducted for NWCL, seven did not report adverse events and none measured the degree of functional and aesthetic impairment, prevention of scarring or QoL. ${ }^{4}$ In a review of 49 trials for OWCL, prevention of scarring was measured in eight studies, QoL in two studies and adverse events in all but three; no study measured the degree of functional or aesthetic impairment. ${ }^{3}$

Despite the lack of patient's views informing the development of CL research to date, there is more widely an increasing consensus that patients should be involved in health research. ${ }^{11}$ Involving patients actively in research has a number of documented benefits: it can lead to higher rates of enrolment and retention and improve its translation into clinical practice. ${ }^{12}$ Furthermore, Domecq et al noted that there is an ethical mandate for the patient participation in research as a manifestation of the 'democratisation' of the research process. ${ }^{11}$

An outcome in the context of a clinical trial refers to what is being measured, that is, any identified result arising from exposure to a causal factor or a health intervention. ${ }^{13}{ }^{14}$ Involving patients in the definition of treatment outcomes, though not frequent practice yet, is recommended ${ }^{131516}$ and already proved effective in identifying outcomes not previously identified by other stakeholders. ${ }^{17} 18$ A Core Outcome Set (COS), consisting of Core Outcomes or Core Outcome Measures, is an agreed minimum set of outcomes that should be measured and reported in clinical trials of a specific disease or trial population. ${ }^{13}$ 17-19 The COMET (Core Outcomes Measures in Effectiveness Trials) initiative ${ }^{20}$ is dedicated to the development of COS, actively involving patients in the process.
Eligibility criteria explicitly define the patient spectrum to be included in/excluded from a clinical trial. Adequate definition and reporting are crucial: overly rigorous eligibility criteria compromise the external validity of a trial, while poorly or too loosely defined eligibility criteria compromise patient safety and make comparison of results between different studies difficult. ${ }^{21}$

Below, we describe the process whereby we constructed a project aimed at understanding perspectives of patients with CL and the protocol that was developed. We wanted to explore patients' experiences with CL through patient interviews to understand their needs and views about their disease as well as requirements and expectations from treatment.

It is important to understand how better to treat CL and to measure treatment effects in a more standardised, meaningful way which takes into account the patient's perspective. This will benefit the generation of evidence and help better define the desired treatment characteristics when developing new medications and medical interventions.

\section{Establishing the patients experiences collaboration in CL (PEC-CL) and protocol development}

This multicentre study is sponsored by the Special Programme for Research \& Training in Tropical Diseases (TDR), hosted by the WHO. Participating experts (local principal investigators (PIs)) were identified through an open competitive call for application published widely on relevant websites ${ }^{22}$ and selected by a review panel. A total of 56 applications were received, of which 22 from NWCL-affected areas and 34 from OWCL-affected areas. The aim was to cover as much as possible the different forms of CL across the world (OWCL and NWCL). Selection criteria for candidates were the geographical origin (corresponding to specific Leishmania species), access to current and prior CL cases, the number and type of CL cases seen per year, the patient spectrum at the site and available resources and collaborators.

TDR originally supported eight participants, although one had to withdraw participation due to a change of institution. A second site in Colombia is supported by the Drugs for Neglected Diseases initiative (DND $i$ ). Of the selected participants, four are men and four women. All of them are CL researchers and/or healthcare professionals (HCPs) with experience with patients with CL. Since capacities for qualitative research in CL-endemic countries are limited, workshops were organised to train investigators and to provide opportunities for collaboration.

TDR convened two 5-day training workshops for the PIs. The first workshop was organised at the beginning for protocol development. In addition, it provided training on planning and conducting qualitative health research with a specific focus on interviewing patients. During this workshop, the topic guide (online supplementary file 1: Interview topic guide) was also developed and tested. The second workshop provided training on analysis 


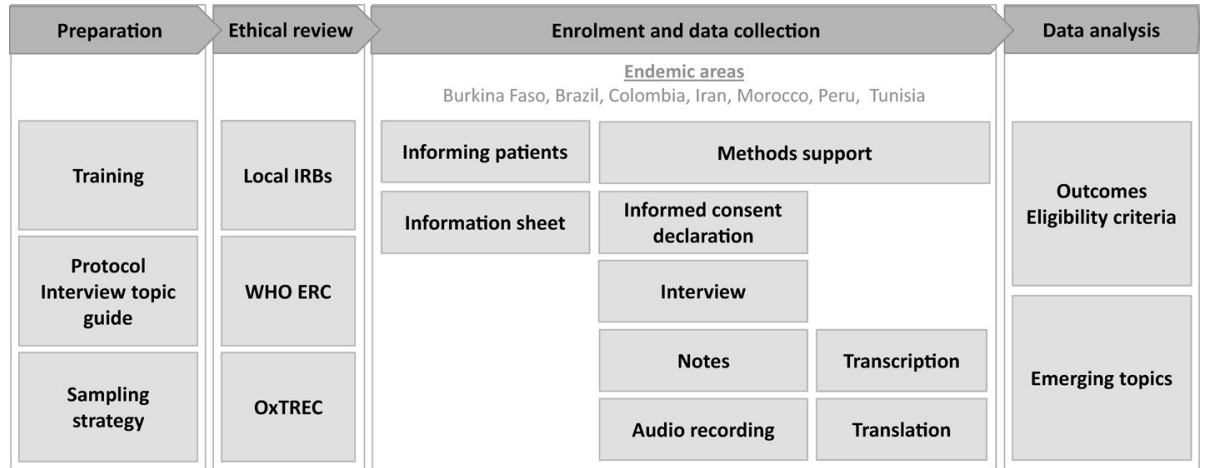

Figure 1 Study workflow. The master protocol including the interview topic guide and the sampling strategy was developed in collaboration during the first workshop. Individual sites were responsible for submission of the master protocol and adapted site-specific protocols to the responsible bodies for ethical clearance. Patient interviews and their transcription and translation are conducted locally. Translated transcripts will be analysed centrally for outcomes and eligibility criteria as well as locally for emerging topics relevant to a specific country or regional context.

of qualitative data using NVivo (QSR Software). Both trainings were held by the Health Experiences Research Group (HERG) at the Nuffield Department of Primary Care Health Sciences at the University of Oxford.

\section{METHODS AND ANALYSIS}

The objectives of this study are:

1. To capture disease experiences of patients with CL in endemic countries by conducting semistructured interviews.

2. To identify eligibility criteria and outcome measures for intervention trials in CL that are important from a patient's perspective through analysis of the interviews. Questions that the study seeks to answer are: Who wants to be treated and under what conditions? and What are the outcomes that patients want to experience or do not want to suffer from?

3. To further explore experiences of patients with CL by in-depth analysis of the interviews to address relevant topics emerging from the data.

\section{Study design}

This is an international qualitative study, coordinated and supported by TDR in collaboration with DND $i$. The study workflow is described in figure 1. Individual, semistructured interviews are conducted at sites in eight endemic countries (Burkina Faso, Brazil, two sites in Colombia, Iran, Morocco, Peru and Tunisia). All sites are using equivalent protocols, collaboratively developed during and after the study workshops. Minor adaptations were made depending on the specific ethics committees' requirements or feedback. The interviews are conducted, transcribed and translated by the local PIs in the endemic regions, who are therefore also referred to as 'interviewers' in this protocol. The study uses pragmatism as research approach, allowing for multiple philosophical worldviews, assumptions and methods for data analysis while focussing on the research question. ${ }^{23-25}$

The reason for choosing individual, semistructured in-depth interviews as opposed to other techniques, for example, focus groups, is that they allow patients to speak free from social dynamics in a group. Answers can be clarified immediately, and the interviewer is able to obtain more in-depth information than in a group context. The aim is to elicit spontaneous, non-biased reports from patients. Questions are open-ended, allowing for further questioning (probing) based on the patient's responses, thereby enabling the interviewer to clarify complex problems associated with a condition and their inter-relationships. ${ }^{26-28}$ Patients are interviewed in their mother tongue (Arabic, Dioula, Farsi, Mòoré, Portuguese and Spanish). The interview topic guide (box 1) for this study, which was developed in collaboration, can be found in online supplementary file 1: Interview topic guide.

\section{Box 1 Topic guides in qualitative research ${ }^{2633}$}

An interview topic guide in qualitative interviews is a checklist of topics to cover during the interview and is as such very different to for example, a patient questionnaire. It includes the issues the investigator wishes to probe and salient points about them.

Different types of questions could explore:

- Descriptions of experiences, for example, Can you start by telling me your story about $C L$ ?

- People's goals, intentions, desires and values, for example, What do you think would be the best treatment for CL? Who do you think should be treated?

- Feelings in order to obtain an understanding of emotional responses, for example, Some people are concerned about scars. How do you feel about scars?

- Knowledge and factual information, for example, Do you know any other treatments for $C L$ ?

- The respondent's previous experiences, for example, How easy was it to get treatment?

As considered particularly important for this study, the interview topic guide (online supplementary file 1: Interview topic guide) will allow the individual interviewer to tailor the order, language, phrases and syntax to the cultural and personal context as much as possible, while ensuring the same topics will be covered across all sites for consistency, thus providing guidance during the interview. 
Box 2 Patient spectrum composition. For patient

recruitment to the multicentre study, maximum variation

along these patient characteristics and parameters is

sought, thus guiding recruitment at individual sites

1. Gender

2. Age

3. Clinical presentation: Anatomical localisation and number of lesions

4. Treatment status:

- Patients currently seeking treatment.

- Patients under treatment.

- Patients who had been treated previously.

5. Patients

- Treated as outpatients.

- Treated as inpatients.

6. Leishmania species: L. major, L. tropica, L. (Viannia) braziliensis, L. (Viannia) panamensis and L. (Viannia) peruviana

7. Treatment modality (Treatment, route of administration and dosing regimen)

8. Endemicity level

Translated interview transcripts will subsequently be analysed using thematic content analysis. ${ }^{27-29}$

\section{Sampling}

Aligned with the qualitative nature of the study, we adopted a non-probability sampling approach, and no sample size calculation was done. This sampling approach is not guided by the idea of random selection and statistical representativeness of the sample for the overall population. However, representativeness of the findings for a patient spectrum that is as broad as possible was considered important for this study despite being rather unusual in the context of qualitative research per se. ${ }^{30-32}$ Therefore, we used a maximum variation sampling approach as a variation of purposive sampling. ${ }^{33}$

For this study, we sought to cover a defined patient spectrum along the characteristics listed in box 2 and to obtain maximum variation within this spectrum. The central assumption was that this would correspond to a maximum variation in patients' experiences. This patient spectrum composition for the entire study, across all sites, was then translated into a sampling approach meaningful for each specific region, taking into consideration the typical patient population at each site (online supplementary file 2: Background parameters informing the sampling strategy). This sampling procedure is based on the assumption that the local PIs, as healthcare providers and researchers, are able to purposively identify patients most informative to the study objectives in the specific cultural context.

We planned for 10 interviews in each of the eight sites. The sample size of 80 patients for the entire study was determined by the available resources and the given timeframe, in the light of the expected complexity of analysis, in particular with regard to the different languages and cultural contexts. Data saturation, describing a point in qualitative data collection when no new or no more relevant information emerges with respect to the theory, ${ }^{32}$ was deemed as unlikely to be achieved and not taken into consideration for this study.

We aim at the majority of interviewed patients reflecting a clinical trial population, since the study focuses on informing clinical trial and product design. Patients with severe comorbidities, MCL or PKDL are excluded.

Only patients above the age of consent in each region are interviewed, that is, 18 years of age in all countries except Burkina Faso, where the legal age of consent is 20 years. The study will however include the perspectives of some children with CL as told by their parents, when being interviewed about their own disease experiences.

\section{Enrolment and data collection}

Data collection is currently ongoing. Participants are identified and invited to participate by the local PIs among patients with a confirmed CL diagnosis (newly diagnosed, on treatment and after treatment). It is made clear to patients that participation in the interviews is voluntary and that participation, or refusal to participate, is completely independent of any current or future treatment or enrolment in clinical trials. Patients do not receive any payment for their participation, but compensation for their time and reimbursement of travel costs, if applicable.

After having been explained the study and being given a Patient information sheet, a separate appointment for the interview is made. At the specified appointment, interviews are conducted after patients have had the opportunity to ask any remaining questions and have given their consent by signature (or appropriate alternative) to the interviewer or a designated person, using an Informed consent declaration.

Documents are presented in languages required by the local Institutional Review Boards (IRBs) and explained to patients if necessary. To obtain consent from participants who are unable to sign, at least one literate witness is chosen from outside the research team. Whenever possible, participants choose the witness(es) themselves.

The interviews are conducted in a healthcare facility, optimally in a separate room to where treatment takes place, or at the patient's home. The setting is chosen balancing its neutrality with giving the patients the opportunity to speak freely and comfortably about sensitive topics without disturbances by, for example, family members. Interviews are conducted in the patients' mother tongues or a language that they are sufficiently comfortable with, roughly following the Topic guide (online supplementary file 1: Interview topic guide). The interviews with a duration of about 1 hour are audio recorded, and the interviewers take notes before, during and after the interview. Patients are free to stop at any time during the interview and to refuse to answer questions they feel uncomfortable with.

Interviews are then transcribed verbatim in the original language and subsequently translated into English. 
Translated transcripts, as well as notes, are also shared for analysis.

\section{Data analysis}

Analysis of qualitative data will follow thematic content analysis methods as described in the literature ${ }^{27-29}$ : Translated transcripts will be analysed and reported along themes. Following the definition, ${ }^{34}$ themes in qualitative research are concepts or propositions that describe and help interpret and explain aspects of the data. For this study, they will be articulated and developed by comparison between and within participants and regions, specifically looking for emerging patterns, similarities and differences. Notes taken before and during the interviews and reflections written up afterwards will inform the process.

For study objectives (2) and (3) and in line with qualitative research, the data will guide the analysis. This twofold analysis approach will provide, in addition to elements relevant for clinical trial design, rich insights into experiences of patients with CL in a low-income and middle-income country context.

In the analysis approach for objective (2), a thematic coding framework will be developed by reading and re-reading some initial interviews and organising sections under different, suitable headings (categories). After familiarising with the data, relevant sections in subsequent interviews will then be organised (coded) with this coding framework, using NVivo 11 software, focussing on the predefined categories outcomes, inclusion and exclusion criteria (eligibility criteria) and related. The coding framework will be subject to continuous update. In a second step, themes will be developed from the data using the 'one sheet of paper' (OSOP) method ${ }^{35}$ or comparable.

Analysis of the pooled interviews for objective (2) will be done centrally by a trained researcher, to ensure consistency of analysis across all sites. An experienced qualitative researcher will provide supervision and, in order to ensure data quality, methodological consistency and transparency, will analyse around $20 \%$ of the interview transcripts in parallel. Discrepancies will be resolved by discussion. As interviews are conducted in the endemic areas, both of these researchers will not have witnessed any of the interviews. This division of labour is rather atypical in the context of qualitative research, where usually the conduct and analysis are being performed by the same person. Challenges are anticipated concerning contextual information, cultural connotations and use of colloquial expressions or dialects, in particular when relating to medical terminology. In order to mitigate this, the interviewers are asked to take detailed notes in order to facilitate analysis. Notes contain as much information as possible, such as on the setting, atmosphere and context of the interview, the main points made by the respondent during this interview, whether they encountered anything surprising or unanticipated during the interview or any problems with the topic guide (eg, wording, missing topics). In addition, subsequent discussion between the interviewers and the researchers analysing the interviews is anticipated during the analysis stage.

In particular with regard to QoL-related outcomes, we anticipate great heterogeneity corresponding to the cultural context. For analysis, we plan to use an established and internationally validated outcome measurement instrument such as the Dermatology Life Quality Index ${ }^{3637}$ as a frame of reference in combination with research approaches for linking outcome measures to established instruments, for example, the International Classification of Functioning, Disability and Health Linking Rules. ${ }^{38}$

Analyses focussing on themes emerging from the data, as described in objective (3), will be performed by the local PIs individually or in groups. An experienced qualitative researcher will provide supervision and support. Due to the highly iterative nature of qualitative analysis, the most suitable method of analysis, informed by the data and the research question, will be reported in more detail together with the study results in subsequent publications. Results are expected to include descriptions and explanations, the development of conceptual definitions and to explore new ideas and inform future research. ${ }^{28}$

\section{Patient and public involvement}

Patients were not involved in the study design, recruitment and conduct, but their experiences and preferences will inform the development of the research questions for analysis as described in objective (3). A lay summary containing the main study results will be provided to the local PIs for dissemination to participants and their communities.

\section{ETHICS AND DISSEMINATION}

Ethical clearance of the protocol used a master protocol as well as site-specific protocols over several submission rounds.

In a first step, a master protocol including supporting documents (Patient information sheet, Informed consent declaration, Interview topic guide and Statement of compliance and confidentiality), developed in collaboration, was submitted for ethical clearance to

- WHO Research Ethics Review Committee (WHO ERC), Geneva, Switzerland, as well as the IRBs and ethics committees (ECs) responsible for the respective sites:

- Comité d'éthique de la Faculté de Médecine et du CHU Hassan II Fes, Fez, Morocco.

- Comité d'éthique Biomédicale de l'Institut Pasteur de Tunis, Institut Pasteur de Tunis, Tunis, Tunisia.

- Comité Institucional de Ética de Investigación en Humanos (CIEIH), Centro Internacional de Entrenamiento e Investigaciones Médicas (CIDEIM), Cali, Colombia.

- Comité d'éthique institutionnel du Centre MURAZ, Bobo-Dioulasso, Burkina Faso. 
- Centro de Pesquisa René Rachou (CPqRR), Fundação Oswaldo Cruz (FIOCRUZ), Minas Gerais, Brazil and Comissão Nacional de Ética em Pesquisa-CONEP, Brazilia, Brazil.

- IRB of Mekelle University, College of Health Sciences, Mekelle, Ethiopia.

- Shiraz University of Medical Sciences (SUMS) Ethics in Research Committee, Shiraz, Iran.

- Comité de Bioética de Investigación en Humanos, Sede de Investigación Universitaria (CBEIH-SIU) of the University of Antioquia, Medellin, Colombia.

- Comité Institucional de Etica para Humanos, Universidad Peruana Cayetano Heredia (CIEH-UPCH), Lima, Perú.

- Oxford Tropical Research Committee (OxTREC), University of Oxford, Oxford, UK.

In a subsequent step, site-specific protocols based on the approved master protocol and containing modifications and translations, as required by the individual IRBs, were submitted for approval.

Of ethical concern for this study is that HCPs assume the role of interviewers and, in most cases, patients being interviewed are aware of this professional background. This 'dual role' could potentially influence the conversation by introducing an undesired relationship (where HCPs would be expected to answer questions and provide medical advice) or even a hierarchy. If necessary, interviewers clarify their role towards patients before or during the interview: that they, as HCPs, are wearing a 'different hat' as interviewers. Britten ${ }^{39}$ recommends ensuring that patients are able to speak freely and not be corrected if they make wrong statements about treatment for example. In cases where patients are found to ask questions concerning medical issues during interviews, interviewers offer to clarify these after the interview and without being audio recorded. Related to that dual role of the investigators in this study, IRBs emphasised the importance of avoiding possible coercion by asking for appropriate measures during recruitment: if the interviewer is treating the patient as HCP, the informed consent is taken by someone else not involved in the patient's treatment. If the interviewer is not treating the patient as HCP, the patient is invited to participate by their treating physician/nurse or someone from the medical team, and the informed consent is then taken by the investigator or someone from the medical team.

Patients will remain anonymous; their names will be replaced by an ID during the transcription and their identity will not be disclosed for the report and any publications. All investigators and designated persons involved in the study have signed a Statement of compliance and confidentiality. Secure electronic and paper-based filing systems were set up to keep study-specific data, which can only be accessed by the research team or designated persons. Electronic recordings (audio files) of the interviews will be stored by the local PIs and will not be forwarded. Transcripts of interviews, translations of transcripts and notes taken during the interviews will be stored locally in a locked file cabinet or electronically. Electronic copies of translations of transcripts and notes will also be shared for analysis, anonymised and password-protected. All study-related records will be kept at the study sites for a minimum of 5 years after the end of the study.

Findings will be reported according to SRQR guidelines ${ }^{40}$ and submitted for publication in peer-reviewed journals and presented at local meetings and larger scientific meetings.

\section{CONCLUSION}

We believe that sharing our experience in designing and executing this project could be useful to other researchers working both on CL and in other areas, as this approach is not disease-specific and has broader applicability.

\section{Author affiliations}

${ }^{1}$ Centre for Tropical Medicine and Global Health, Nuffield Department of Medicine, University of Oxford, Oxford, UK

${ }^{2}$ Department of Epidemiology, Center for Public Health, Medical University of Vienna, Vienna, Austria

${ }^{3}$ Drugs for Neglected Diseases Initiative (DNDi), Geneva, Switzerland

${ }^{4}$ National School of Public Health, Rabat, Morocco

${ }^{5}$ Department of Public Health, Institute of Tropical Medicine, Antwerp, Belgium

${ }^{6}$ Institut Pasteur de Tunis, Tunis, Tunisia

${ }^{7}$ Department of Family and Community Medicine, College of Medicine and Medical Sciences, Arabian Gulf University (AGU), Manama, Bahrain

${ }^{8}$ Centro Internacional de Entrenamiento de Investigaciones Médicas (CIDEIM), Cali, Colombia

${ }^{9} \mathrm{EDCTP} / T D R$ Fellow, European Vaccine Initiative (EVI), Heidelberg, Germany

${ }^{10}$ Centre MURAZ, Bobo-Dioulasso, Burkina Faso

${ }^{11}$ Centro de Pesquisa René Rachou (CPqRR), Fundação Oswaldo Cruz (FIOCRUZ), Minas Gerais, Brazil

${ }^{12}$ Molecular Dermatology Research Center, Shiraz University of Medical Sciences, Shiraz, Iran

${ }^{13}$ Ayder Referral Hospital, College of Health Sciences, Mekelle University, Mekelle, Ethiopia

${ }^{14}$ Programa de Estudio y Control de Enfermedades Tropicales (PECET), Universidad de Antioquia, Medellín, Colombia

${ }^{15}$ Instituto de Medicina Tropical Alexander von Humboldt, Universidad Peruana Cayetano Heredia, Lima, Peru

${ }^{16}$ Departamento de Enfermedades Infecciosas, Dermatológicas y Tropicales, Hospital Cayetano Heredia, Lima, Peru

${ }^{17}$ Special Programme for Research \& Training in Tropical Diseases (WHO/TDR), Geneva, Switzerland

Acknowledgements We would like to thank Dr Christine Halleux for her support with ethical review, Abdul Masoudi and Lauren Whelan for their administrative support and the HERG team at the Nuffield Department of Primary Care Health Sciences at the University of Oxford for the great training in qualitative research during two workshops. We are particularly grateful to all the patients with $\mathrm{CL}$ who have contributed so far to the study.

Contributors PO, BA, ACE, LLC and EP conceived the study and ACE was responsible for drafting the initial study protocol. All authors were involved in finalising the protocol and designing the interview topic guide and the sampling strategy. They have also agreed on the final version of this article, having been involved in drafting or critically revising draft versions of the manuscript.

Funding This work was supported by grants from the Special Programme for Research and Training in Tropical Diseases (TDR) and the Drugs for Neglected Diseases initiative (DNDi). ACE is a recipient of a DOC-fFORTE fellowship of the Austrian Academy of Sciences at the Nuffield Department of Medicine, University of Oxford.

Disclaimer The opinions expressed in this paper are those of the authors and may not reflect those of their employing organisations. PO is a staff member of the WHO; 
the authors alone are responsible for the views expressed in this publication and they do not necessarily represent the decisions, policy or views of the WHO.

Competing interests None declared.

Patient consent Not required.

Ethics approval World Health Organization Research Ethics Review Committee (WHO ERC), Geneva, Switzerland as well as the IRBs and ethics committees (ECs) responsible for the respective sites: Comité d'éthique de la Faculté de Médecine et du CHU Hassan II Fes, Fez, Morocco; Comité d'éthique Biomédicale de I'Institut Pasteur de Tunis, Institut Pasteur de Tunis, Tunis, Tunisia; Comité Institucional de Ética de Investigación en Humanos (CIEIH), Centro Internacional de Entrenamiento e Investigaciones Médicas (CIDEIM), Cali, Colombia; Comité d'éthique institutionnel du Centre MURAZ, Bobo-Dioulasso, Burkina Faso; Centro de Pesquisa René Rachou (CPqRR), Fundação Oswaldo Cruz (FIOCRUZ), Minas Gerais, Brazil; Comissão Nacional de Ética em Pesquisa_CONEP, Brazilia, Brazil; IRB of Mekelle University, College of Health Sciences, Mekelle, Ethiopia; Shiraz University of Medical Sciences (SUMS) Ethics in Research Committee, Shiraz, Iran; Comité de Bioética de Investigación en Humanos, Sede de Investigación Universitaria (CBEIH-SIU) of the University of Antioquia, Medellin, Colombia; Comité Institucional de Etica para Humanos, Universidad Peruana Cayetano Heredia (CIEH—UPCH), Lima, Perú; Oxford Tropical Research Committee (OxTREC), University of Oxford, Oxford, UK.

Provenance and peer review Not commissioned; externally peer reviewed.

Open access This is an open access article distributed in accordance with the Creative Commons Attribution Non Commercial (CC BY-NC 4.0) license, which permits others to distribute, remix, adapt, build upon this work non-commercially, and license their derivative works on different terms, provided the original work is properly cited and the use is non-commercial. See: http://creativecommons.org/ licenses/by-nc/4.0/

(c) Article author(s) (or their employer(s) unless otherwise stated in the text of the article) 2018. All rights reserved. No commercial use is permitted unless otherwise expressly granted.

\section{REFERENCES}

1. Alvar J, Vélez ID, Bern C, et al. Leishmaniasis worldwide and globa estimates of its incidence. PLoS One 2012;7:e35671.

2. World Health Organization. Leishmaniasis fact sheet. 2018. http:// www.who.int/mediacentre/factsheets/fs375/en/ (accessed $18 \mathrm{Apr}$ 2018)

3. González U, Pinart M, Reveiz L, et al. Interventions for old world cutaneous leishmaniasis. Cochrane database of systematic reviews. 30: John Wiley \& Sons, Ltd, 2008. (accessed 22 Apr 2014).

4. González U, Pinart M, Rengifo-Pardo M, et al. Interventions for American cutaneous and mucocutaneous leishmaniasis. Cochrane database of systematic reviews. 180: John Wiley \& Sons, Ltd, 2009. (accessed 22 Apr 2014).

5. González U, Pinart M, Reveiz L, et al. Designing and reporting clinical trials on treatments for cutaneous leishmaniasis. Clin Infect Dis 2010;51:409-19.

6. Monge-Maillo B, López-Vélez R. Therapeutic options for old world cutaneous leishmaniasis and new world cutaneous and mucocutaneous leishmaniasis. Drugs 2013;73:1889-920.

7. Reveiz L, Maia-Elkhoury AN, Nicholls RS, et al. Interventions for American cutaneous and mucocutaneous leishmaniasis: a systematic review update. PLoS One 2013;8:e61843.

8. López-Carvajal L, Vélez I, Arbeláez MP, et al. Eligibility criteria and outcome measures adopted in clinical trials of treatments of cutaneous leishmaniasis: systematic literature review covering the period 1991-2015. Trop Med Int Health 2018;23:448-75.

9. Olliaro $P$, Vaillant $M$, Arana $B$, et al. Methodology of clinical trials aimed at assessing interventions for cutaneous leishmaniasis. PLoS Negl Trop Dis 2013;7:e2130.

10. Olliaro P, Grogl M, Boni M, et al. Harmonized clinical trial methodologies for localized cutaneous leishmaniasis and potential for extensive network with capacities for clinical evaluation. PLoS Negl Trop Dis 2018;12:e0006141.

11. Domecq JP, Prutsky G, Elraiyah T, et al. Patient engagement in research: a systematic review. BMC Health Serv Res 2014;14:89.
12. Sullivan $P$, Goldmann D. The promise of comparative effectiveness research. JAMA 2011;305:400-1.

13. Boers M, Kirwan JR, Wells G, et al. Developing core outcome measurement sets for clinical trials: OMERACT filter 2.0. J Clin Epidemiol 2014;67:745-53.

14. Prinsen CA, Vohra S, Rose MR, et al. Core Outcome Measures in Effectiveness Trials (COMET) initiative: protocol for an international Delphi study to achieve consensus on how to select outcome measurement instruments for outcomes included in a 'core outcome set'. Trials 2014;15:247.

15. Sinha IP, Smyth RL, Williamson PR. Using the Delphi technique to determine which outcomes to measure in clinical trials: recommendations for the future based on a systematic review of existing studies. PLoS Med 2011;8:e1000393.

16. Tugwell $P$, Boers $M$, Brooks $P$, et al. OMERACT: an international initiative to improve outcome measurement in rheumatology. Trials 2007;8:38.

17. Williamson PR, Altman DG, Blazeby JM, et al. Developing core outcome sets for clinical trials: issues to consider. Trials 2012;13:132.

18. Williamson P, Altman D, Blazeby J, et al. Driving up the quality and relevance of research through the use of agreed core outcomes. $J$ Health Serv Res Policy 2012;17:1-2.

19. Clarke M. Standardising outcomes for clinical trials and systematic reviews. Trials 2007;8:39.

20. COMET. Home :: Core Outcome Measures in Effectiveness Trials Initiative (COMET). 2017 http://www.comet-initiative.org/ (accessed 15 Nov 2017).

21. Rothwell PM. Factors that can affect the external validity of randomised controlled trials. PLoS Clin Trials 2006;1:e9.

22. WHO/TDR. Call for expression of interest. 2015 http://www.who.int/ tdr/grants/CL_Call_for_Expression_of_Interest.pdf?ua=1 (accessed $18 \mathrm{Jul} 2017$ )

23. Creswell JW. Research design: qualitative, quantitative, and mixed methods approaches. 4th ed: SAGE Publications, 2013.

24. Creswell JW, Plano Clark VL. Designing and conducting mixed methods research. 2nd ed: SAGE Publications, 2011.

25. Cherryholmes $\mathrm{CH}$. Notes on pragmatism and scientific realism. Educ Res 1992;21:13-17.

26. Patton MQ. Qualitative evaluation and research methods: SAGE Publications, 1990

27. Braun V, Clarke V. Using thematic analysis in psychology. Qual Res Psychol 2006;3:77-101.

28. Green J, Thorogood N. Qualitative methods for health research. 3rd ed: SAGE Publications, 2014

29. Pope C. Analysing qualitative data. BMJ 2000;320:114-6.

30. Gobo G. Sampling, representativeness and generalizability. In: Seale C, Gobo G, Gubrium JF, eds. Qualitative research practice: SAGE Publications, 2004:405-27.

31. Mays N, Pope C. Rigour and qualitative research. BMJ 1995;311:109-12.

32. Neuman WL. Social research methods: qualitative and quantitative approaches. 7th ed: Pearson, 2013.

33. Bowling A. Research methods in health: investigating health and health services: McGraw-Hill International, 2009.

34. Gale NK, Heath G, Cameron E, et al. Using the framework method for the analysis of qualitative data in multi-disciplinary health research. BMC Med Res Methodol 2013;13:117.

35. Ziebland S, McPherson A. Making sense of qualitative data analysis: an introduction with illustrations from DIPEx (personal experiences of health and illness). Med Educ 2006;40:405-14

36. Finlay AY, Khan GK. Dermatology Life Quality Index (DLQI)--a simple practical measure for routine clinical use. Clin Exp Dermatol 1994;19:210-6.

37. Bennis I, De Brouwere V, Belrhiti Z, et al. Psychosocial burden of localised cutaneous Leishmaniasis: a scoping review. BMC Public Health 2018;18:358.

38. Heerkens $\mathrm{Y}$, de Brouwer C, Engels J, et al. ICF manual unit 2: introduction to ICF linking rules and ICF core sets. In: Brouwer CPMde, Heerkens YF, Kant IJ, eds. Occupational health from a biopsychosocial perspective: an evidence based approach. mediview, 2015:1-24.

39. Britten N. Qualitative interviews in medical research. BMJ 1995;311:251-3.

40. O'Brien BC, Harris IB, Beckman TJ, et al. Standards for reporting qualitative research: a synthesis of recommendations. Acad Med 2014;89:1245-51. 